\title{
Fluid substitution in heavy oil rocks
}

\section{Dina Makarynska and Boris Gurevich*, Curtin University of Technology and CSIRO}

\section{Summary}

Heavy oils are defined as having high densities and extremely high viscosities. Due to their viscoelastic behavior the traditional rock physics based on Gassmann theory becomes inapplicable. In this paper, we use effective-medium approach known as coherent potential approximation or CPA as an alternative fluid substitution scheme for rocks saturated with viscoelastic fluids. Such rocks are modelled as solids with elliptical fluid inclusions when fluid concentration is small and as suspensions of solid particles in the fluid when the solid concentration is small. This approach is consistent with concepts of percolation and critical porosity, and allows one to model both sandstones and unconsolidated sands.

We test the approach against known solutions. First, we apply CPA to fluid-solid mixtures and compare the obtained estimates with Gassmann results. Second, we compare CPA predictions for solid-solid mixtures with numerical simulations. Good match between the results confirms the applicability of the CPA scheme. We extend the scheme to predict the effective frequency- and temperature-dependent properties of heavy oil rocks. CPA scheme reproduces frequency-dependent attenuation and dispersion which are qualitatively consistent with laboratory measurements and numerical simulations. This confirms that the proposed scheme provides realistic estimates of the properties of rocks saturated with heavy oil.

\section{Introduction}

Heavy oil reserves account for more than 6 trillion barrels in place worldwide-triple the world reserves of conventional oil and gas (Batzle et. al, 2006; Behura et. al, 2007). However, high viscosity of heavy oils makes them difficult or impossible to produce using conventional technology. A variety of methods, currently employed by the industry, aim at lowering the viscosity by injection of heat or chemical solvents. Thermal recovery methods proved to be the most efficient in heavy oil production with recovery rates as high as $80 \%$. Injection of heat and production change the already complex physical properties of the heavy oil reservoirs such as seismic velocities, density, and attenuation. Tracking these changes by timelapse (or 4-D) seismic techniques can considerably improve the recovery efficiency. An appropriate rock physics modelling is critical for quantitative interpretation of the changes in seismic response due to alterations in reservoir properties during production.

Seismic fluid substitution modelling using Gassmann equation has become routine in the analysis and interpretation of seismic velocities and amplitudes. Given pore, frame, and fluid properties of a rock, it can predict the bulk modulus of a rock under quasi-static conditions. The corresponding dynamic moduli can be obtained from Biot's equations of poroelasticity, which is an extension of Gassmann theory to finite frequencies. However, neither classical Gassmann nor Biot's theory is applicable if the pore filling material is viscoelastic. The reason for this is that Gassmann equation is based on the Pascal law, which states that, in the absence of body forces, fluid pressure is the same throughout the pore space. This law is not applicable to any viscoelastic media. According to laboratory measurements (Batzle et al., 2006) heavy oils exhibit viscoelastic behavior, which is strongly frequencyand temperature- dependent. The oil that at room temperature in a wide range of frequencies behaves as a nearly elastic solid can act as a Newtonian fluid at temperatures above $200^{\circ} \mathrm{C}$. Thus, Gassmann and Biot's theories cannot be applied to heavy oil rocks at high frequencies or low temperatures.

In this paper, we propose an alternative fluid substitution method based on one particular equivalentmedium approach known as coherent potential approximation (CPA) (Berryman, 1980). We show that the method provides reliable estimates of effective frequencyand temperature-dependent properties of heavy oil rocks and can be used for practical applications.

\section{Fluid substitution methodology}

CPA, which was originally proposed in quantum mechanics, is one of the popular mixing laws of the theory of composites. It was introduced by Berryman (1980) as a self-consistent version of the Kuster-Toksoz model. The method uses the concepts of elastic-wave scattering theory for the deformation of isotropic inclusions and approximates the interaction of the inclusions by replacing the background medium with as-yet-unknown effective medium. There is no one constituent acting as a host to all the others, but a more abundant constituent is the loadbearing one. This agrees with the concepts of percolation and critical porosity. CPA accounts for geometric details of the shapes and spatial distributions of the components. This is an attractive feature because seismic properties of a rock are more affected by the pore shape than by porosity. For example, a low-porosity rock with flat, low-aspect ratio pores may have lower seismic velocities than a highporosity rock with spherical, high-aspect ratio pores because flat pores are much more compressible than spherical pores.

CPA can be used for calculating the effect of fluid substitution on seismic properties of porous rocks. It 


\section{Fluid substitution in heavy oil rocks}

has no limitations with respect to the fluid viscosity. Thus, it can be applied to viscous fluids. CPA calculates the effective properties of a rock using the known properties of the solid matrix, the pore fluid, and the pore aspect ratio. The implicit CPA formulas for bulk $K$ and shear $\mu$ moduli of a two component rock (one solid phase, one fluid phase) are:

$K_{n+1}=\left(\phi K_{f} P_{n}^{f}+(1-\phi) K_{s} P_{n}^{s}\right) /\left(\phi P_{n}^{f}+(1-\phi) P_{n}^{s}\right)$

$\mu_{n+1}=\left(\phi \mu_{f} Q_{n}^{f}+(1-\phi) \mu_{s} Q_{n}^{s}\right) /\left(\phi Q_{n}^{f}+(1-\phi) Q_{n}{ }^{s}\right)$,

where $\phi$ is porosity, $K_{f}$ and $\mu_{f}$ are the bulk and shear moduli of the pore fluid, $K_{s}$ and $\mu_{s}$ are the bulk and shear moduli of the matrix (grain), and $P_{n}$ and $Q_{n}$ are invariants of the socalled $\mathrm{Wu}$ tensor (for ellipsoidal inclusions of arbitrary aspect ratio $\alpha$ ), which are evaluated using $K_{n}$ and $\mu_{n}$.

Equations (1) and (2) allow estimating effective elastic moduli of a saturated rock as well as its drained moduli $\left(K_{d r y}, \mu_{d r y}\right)$. The equations are coupled and must be solved by iteration process requiring the first guess of $K_{I}$ and $\mu_{l}$, which can be calculated by Voigt-Reuss-Hill average. In general, CPA application is a two-part process. We first determine the pore aspect ratio $\alpha$, after which we calculate the bulk modulus of the rock saturated with any desired fluid. In practice, $\alpha$ is hard to quantify as pore shape varies in wide ranges in sedimentary rocks. Statistical relationships between porosity and seismic properties need to be established for each facies in a reservoir. On the other hand, $K_{d r y}$ may be known either from lab measurements, direct calculations from log data or numerical simulations. In such case $\alpha$ can be estimated by the inverted CPA scheme for $K_{d r y}$ simply setting the fluid moduli to zero. Then fluid substitution can be performed for any fluid using the direct CPA scheme.

CPA fluid substitution scheme is not limited to monomineralic rocks saturated with one fluid only. It can be applied to multiphase solids saturated with a mixture of fluids. It can also account for different shape of inclusions such as spheres, needles, disks and penny shaped cracks.

\section{Validation}

In general, viscoelastic behavior may be imagined as a spectrum with viscous flow as one limiting case and elastic deformation the other extreme case, with varying combinations of the two spread over the range between. Hence, before applying CPA fluid substitution scheme to heavy oils, it is reasonable to verify it for the limiting cases such as (1) Newtonian fluid pore fill and (2) solid pore fill by a comparison with known exact solutions. As can be seen from Figure 1, the CPA predictions for quartz/water mixtures agree reasonably well with the Krief estimates and lie between the rigorous $\mathrm{H}-\mathrm{S}$ bounds for a wide range of porosities and pore aspect ratios. The maximum deviation of $K-K_{d r y}$ from Gassmann estimates is
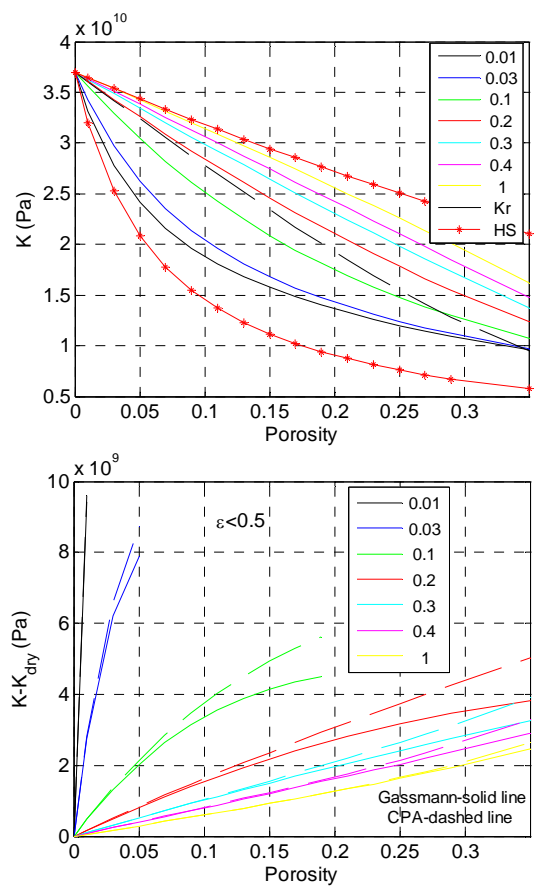

Figure 1. Saturated bulk modulus (upper) and difference between the saturated bulk and dry moduli (lower) vs. porosity for different aspect ratios (indicated by color). Fluid pore fill.

about $1 \mathrm{GPa}(\alpha=0.1)$ for realistic crack densities $(\varepsilon<0.5)$. For the porosity $(\phi=0.25)$ and aspect ratios of interest in this paper $(\alpha=0.3-0.4)$ we observe much better agreement with Gassmann. The deviation is about $0.2 \mathrm{GPa}$.

In Figure 2, we compare the CPA predictions with static finite element (FEM) numerical simulations (Arns et. al, 2002) and with Gassmann estimates. We apply our numerical simulations to a 3D $\left(200^{3}\right)$ image (tomogram) of a real rock with $\phi=0.26$. The corresponding CPA estimates are obtained using inversely derived $\alpha$ from the numerically estimated $K_{d r y}$. CPA predictions are in excellent agreement with the numerical results and lie between the H-S bounds.

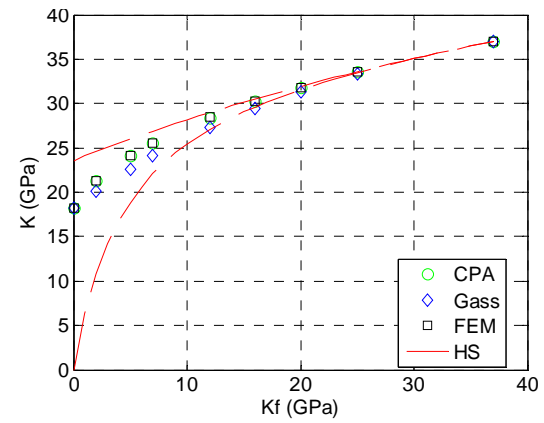

Figure 2. Bulk modulus of a solid/solid mixture vs. moduli of pore fill: CPA, Gassmann, FEM, and HS. 


\section{Fluid substitution in heavy oil rocks}

In contrast, Gassmann estimates deviate significantly from the simulations and do not always satisfy the H-S bounds. In the next set of numerical experiments with the same tomogram, we keep $K$ constant $(K=7 \mathrm{GPa})$ and vary $\mu_{f}$ (Figure 3 ). As in the previous case, the CPA estimates are in a good agreement with the numerical data, while the Gassmann estimates deviate from the numerical results by more than 1.6 GPA.

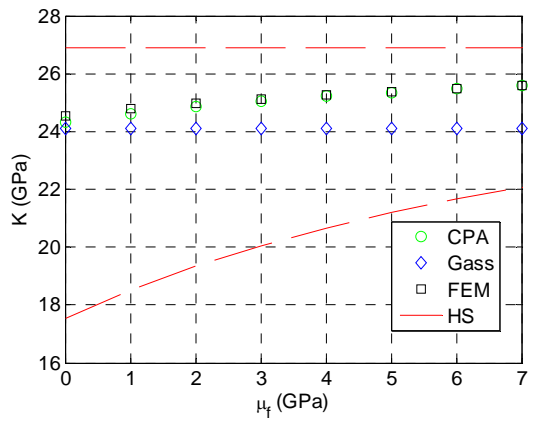

Figure 3. Bulk modulus of a solid/solid mixture vs. shear modulus of pore fill: $K_{s}=$ const $=7 \mathrm{GPa}, \alpha=0.39$ obtained by inverse CPA for $K_{d r y}$.

Successful application of the method to fluid saturated rocks as well as solid-solid mixtures proves that the CPA scheme is reliable and can be applied to heavy oil rocks.

\section{Application to heavy oil}

According to laboratory measurements (Behura et al., 2007) the behavior of heavy oil within the rock dominates the mechanical response of the rock with changing temperature and frequency. Shear-wave properties are the most sensitive to the changes in the heavy oil reservoir due to viscoelastic rheology of the oil. For example, for a given temperature the shear modulus of heavy oil increases with frequency.

We can account for the effects of frequency dispersion and temperature dependency in CPA fluid substitution scheme by employing a generalized Maxwell model of viscoelastic medium (Gurevich et al., 2007). The model is consistent with laboratory data of Batzle et al. (2006) and satisfies the casuality and dissipativity conditions. It also employs an empirical relationship for temperature dependency based on the assumption that the relaxation time is a function of temperature.

We simply employ the generalized Maxwell model to compute the complex shear modulus of heavy oil and use it in the CPA fluid substitution scheme instead of $\mu_{f}$. Frequency dispersion usually leads to significant seismic attenuation within the fluid phase. In our analysis, we use the standard definition of dimensionless attenuation (inverse quality factor) $1 / Q$ for compressional and shear waves.
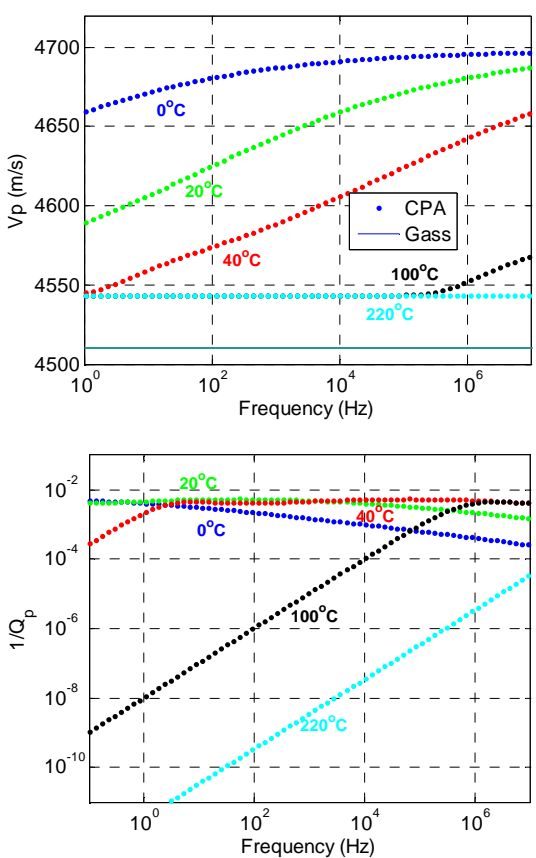

Figure 4. P-wave velocity (upper) and attenuation (lower) in oil/solid mixture vs. frequency and temperature. Comparison with Gassmann predictions (upper) computed using $K_{d r y}$ and $\mu_{d r y}$ obtained by CPA with empty pores.

Figure 4 shows the CPA results for $\mathrm{P}$-wave in a heavy oil sand with $\alpha=0.39$. The CPA results for P-wave demonstrate significant frequency and temperature dependence. We observe the velocity dispersion for the temperatures below $100^{\circ} \mathrm{C}$. At low temperatures, the oil approaches its glass point and begins to act as a solid. We can see that at $T=40^{\circ} \mathrm{C}$ the shear properties are in transition, that is at high frequencies the oil is still effectively a solid, however, at seismic frequencies it can go through shear relaxation and act as a fluid with no shear modulus. At higher temperatures the oil acts as a Newtonian liquid, hence, $V_{p}$ becomes weakly dependent on frequency.

We also compute Gassmann estimates using the dry bulk and shear moduli calculated by CPA with empty pores. As expected, velocity decreases with increasing temperature towards Gassmann solution We see that the CPA predictions are quite satisfactory, deviating from Gassmann by about $35 \mathrm{~m} / \mathrm{s}$ when fluid is Newtonian (e.g. frequencies are low or temperatures are high).

Figure 4 also shows the dependence of inverse quality factor (attenuation) of heavy oil rock on temperature and frequency. In the lower viscosity range (high temperatures, low frequencies) the lowest values of the inverse quality factor demonstrate more fluid-like behavior of heavy oil. We can see that the attenuation drops 


\section{Fluid substitution in heavy oil rocks}

dramatically as the temperature is increased. At low temperatures (high frequencies), the attenuation is dominated by the classical viscoelastic absorption due to shear stiffening effect of the viscous pore fluid. The shift in the attenuation peak toward lower frequencies with decreasing temperatures occurs due to competing effects of decreasing frequency (longer flow time) and decreasing temperature (higher viscosity).
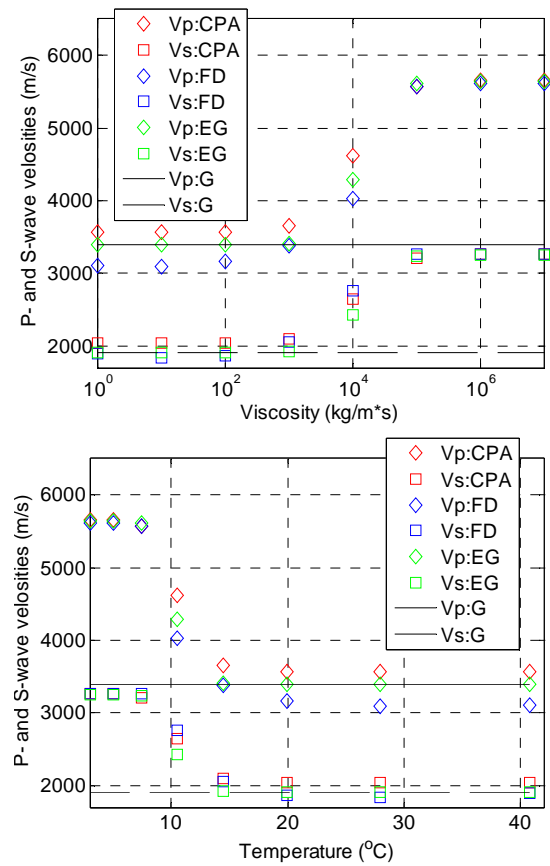

Figure 5. P- and S-wave velocities vs. viscosity (left) and temperature (right): predictions by CPA, numerical simulations (FD), extended Gassmann (EG), and classical Gassmann (G).

In Figure 5, we compare the CPA predictions with finite-difference (FD) elastodynamic simulations (Saenger et al., 2005) as well as with data produced by viscoelastic extension of Gassmann theory (EG) (Ciz et al., 2007). The FD numerical code uses a displacement-stress rotated staggered finite-difference grid to solve the elastodynamic wave equation. We also show in this figure the estimates obtained by Gassmann equation (G). We see that at higher viscosities $\eta>10000 \mathrm{~kg} / \mathrm{ms}$ and $T<10^{\circ} \mathrm{C}$, the results almost coincide, except $\mathrm{G}$ estimates, which deviate by almost $2000 \mathrm{~m} / \mathrm{s}$ for P-wave velocity. The largest mismatch between the data is observed for $\eta=10000$ $\left(T=10^{\circ} \mathrm{C}\right)$ for $\mathrm{P}$-wave when oil is in transition between viscoelastic and viscous state. We can see that the CPA estimates are more consistent with the EG results. At $\eta<10000 \mathrm{~kg} / \mathrm{ms}$ and $T>10^{\circ} \mathrm{C}$ the oil is in liquid state and all data tend to follow the Gassmann solution. However, some discrepancies between FD and G data are observed for low viscosity. This discrepancy caused by insufficient grid sampling of the viscous boundary layer near the solid/fluid interface. A good agreement between the results suggests that the CPA approach can be used as an alternative fluid substitution scheme.

In our predictions, we have used a symmetric CPA scheme, where both types of constituents (quartz and heavy oil) were assumed to have the same geometrical shape (oblate spheroid). However, a combination of geometrical shapes describing more properly the geometry of grains and pore space might further improve the results.

\section{Conclusions}

To account for the viscoelastic properties of heavy oils we have proposed an approximate fluid substitution scheme based on self-consistent mixing method known as Coherent Potential Approximation (CPA). First, CPA equations are inverted to obtain pore effective aspect ratio from dry moduli. Then forward CPA is used to obtain saturated moduli.

We have compared the CPA estimates for fluid/solid, solid/solid, viscoelastic material/solid mixtures with analytical and numerical solutions. For high temperatures or low frequencies, when the pore fill can be considered Newtonian, CPA gives results approximately consistent with Gassmann equations. In the other limit, when the pore fill is nearly elastic, the results show good agreement with numerical predictions and are consistent with rigorous $\mathrm{H}-\mathrm{S}$ bounds. At the intermediate temperatures CPA reproduces frequency-dependent attenuation and dispersion which is consistent with numerical data as well as results produced by viscoelastic extension of Gassmann. This confirms that the proposed scheme provides realistic estimates of the properties of heavy oil rocks and can be used as an alternative fluid substitution approach for rocks saturated with viscoelastic fluids.

\section{Acknowledgements}

This work was kindly supported by the Centre of Excellence for Exploration and Production Geophysics, CSIRO Division of Petroleum Resources, Curtin Reservoir Geophysics Consortium, APA Postgraduate Scholarship, and the Australian Partnership for Advanced Computing. 